\title{
Generalised Fuzzy Aggregation in Estimating the Risk of Desertification of a burned forest
}

\author{
K. R. Sasikala and M. Petrou \\ School of Electronic Engineering, Information Technology and Mathematics \\ University of Surrey, Guildford, GU2 5XH, U.K. \\ Fax:+44 - 1483 - 534139 \\ Tel:+44 - 1483 - 259801
}

\begin{abstract}
This paper investigates two aspects of using Fuzzy Logic with a GIS: The use of non-conventional aggregation operators for conjunctive and disjunctive reasoning and the use of weights when combining aggregates of different significance to the classification. Two methods of weighting are used, weights as powers of the membership functions, and the novel concept of membership functions that take values greater than 1 . The two approaches are investigated in conjunction with 121 possible combinations of aggregation operators that are used to reason in the disjunctive and conjunctive level of the problem of assessing the risk of desertification after a forest fire.
\end{abstract}

Keywords: Fuzzy Sets, GIS, Aggregation, Relative importance of aggregates.

\section{Introduction}

In Geography and related sciences, a GIS package is often used for combining information from various sources for the ultimate purpose of region classification. Most of the commercially available GIS packages tend to simply superimpose the various information layers in a heuristic way. Apart from the obvious problem that different factors may combine in a non-linear way to influence the output classification, there is another major drawback of this approach: the information obtained from each layer may be inaccurate or uncertain to various degrees and this uncertainty should be taken into consideration when the various items of information are combined. In order to preserve the flexibility offered by a GIS in storing and retrieving geographical information, while taking into consideration both the way the different factors influence the final classification and the uncertainty in the value or class of each of them, we propose the use of a GIS system with reasoning mechanisms that can cope with the above requirements.

We shall present our ideas in the context of the problem of burned forest management. In particular, we are interested in ranking burned forests in order of risk of desertification. There is a lot of work done on mapping and monitoring forest areas [1] [24] [8] [7] 
[17], assessment of vegetation change [18], fire risk assessment [22] [9] [15] and restoration of burned areas and vegetation recovery [3] [33] [25] [16]. Although the above approaches use traditional Remote Sensing and GIS techniques for spatial data integration, there are plenty of other examples where uncertainty in the available information is taken into consideration. The list of mechanisms, which have been proposed in Geographical and Geological applications, that can handle uncertainty, includes Bayesian networks [6] [21] [29], neural networks and genetic algorithms [41] [30], [31], Dempster-Shafer theory [23] [21] [19] and fuzzy logic [4] [27]. In [21], Lee and Richards discuss both a conventional statistical method and a Dempster-Shafer method for multisource data analysis and suggest that a combination of the two approaches may be well suited when there are both numerical and non-numerical data. In [6], Bonham-Carter et al use Bayesian statistics to combine various geological factors in finding areas favourable for gold exploration. Moon in [23] uses a Dempster-Shafer method to deal with incomplete information and also to integrate geophysical and geological data sets of different resolution. In [41], Zhou and Civco use an evolutionary learning algorithm in a Neural Network as a replacement and an improvement over traditional methods of GIS for suitability analysis in order to find all areas suitable for the location of a light manufacturing plant.

Of all the above soft-computing techniques, by far the most flexible one, both in terms of implementation and intuitive understanding, is fuzzy logic [40]. In the problem we are interested in, i.e., the assessment of the degree of risk of desertification of areas affected by fire, the variables involved are fuzzy with a mixture of classes. Thus, fuzzy logic has been chosen as the main reasoning mechanism. The decision making process involves implementing rules that contain linguistic variables or fuzzy sets. This demands a procedure for aggregation of fuzzy sets. There are several publications available on fuzzy aggregation operators, of which a few notable ones are [20] [2] [13] [38] [34] [14] [12] [5] [10] [39] [32] [11]. In these papers, the properties of various aggregation operators are stated. With a few exceptions, in most applications of fuzzy logic to the Environmental Sciences, the classical $\min$ and $\max$ aggregation operators are used, while the other options remain largely unexplored. (For example, Binaghi and Rampini [4] use fuzzy aggregation in fire risk determination, but mainly concentrate on the mean aggregation operator as an alternative to $\max$ and $\mathrm{min}$ ). Choosing the right operator or sets of operators for a problem may not be straightforward. To deal with the example problem we use in this paper, we need a combination of two operators. If we take into consideration the nature of the various operators, we end up with 121 possibilities. We advocate here a training-based approach, during which the most appropriate set of operators is identified.

Another important issue that has to be considered, is the relative importance of the various factors that are aggregated by the fuzzy logic mechanism. There have been a few publications that say how relative importances of combined factors could be incorporated in fuzzy reasoning. Of these, the most notable ones are by Yager [34] [35] [36] [37]. In this paper, we introduce the novel idea of assigning relative importance to the aggregated factors by allowing the membership functions to take values greater than 1 . It must be stressed that this is not the same as scaling the memberships functions with the help of weights, because several of the aggregation operators used are non-linear and scaling their arguments is not the same as allowing memberships functions greater than 1 . For this purpose, we also propose generalised forms of these operators where their input variables are 
allowed to take values greater than 1 . The properties of these generalised operators are discussed in detail in [26]. The maximum value of each membership function is treated as a parameter that is determined with the help of the training data. This approach is compared with Yager's basic approach, namely that of membership functions raised to some power.

Briefly, the purpose of this paper is dual:

First, to explore a new mechanism for taking into consideration the relative importance of the various factors that influence a decision, and second to investigate this mechanism in conjunction with the various rules that have been proposed for evidence aggregation and in the context of a specific application. Hence, this work is aimed at improving a conventional GIS-based system in a decision making process that involves spatial data analysis. This improvement will be exemplified by experimental comparison with a simple GIS rule-based system.

Section-II of the paper introduces the problem. Section-III gives a brief overview of fuzzy techniques including the properties of a few aggregation operators. Section-IV brings out the issues related to the relative importances of the aggregates, reviews Yager's work and also describes our approach. Section-V describes the various experiments performed. Section-VI discusses the results obtained, justifies why the choice of aggregation operators and inclusion of relative importances matter in a decision making problem, and summarizes briefly the main points that have emerged as the outcome of the experiments and analysis done.

\section{Problem Background}

Forest fires are a major concern throughout the world and though some times a burned forest regenerates on its own in due course, many times the affected area becomes arid if left uncared for. Since total afforestation is virtually impractical, timely and accurate information on the sites that should have priority for reforestation could go a long way in an efficient planning of resource allocation. This could be accomplished by using an efficient reasoning model which could handle fuzzy data as well.

Since the uncertainty involved in the problem is mainly due to a mixture of classes and not due to randomness, usage of partial membership functions is more appropriate than probabilistic approaches. The data that are used in this study pertain to a few sites in Attica, Greece, which have been chosen in such a way that they represent maximum site variability.

The parameters that influence the risk of desertification as given by some experts are shown in figure 1. From this figure, it can be seen that the ultimate variables involved in the study are Ground Slope, Rock Permeability, Soil Depth and Aspect. It is true that there are several other factors that influence desertification, but over the whole study area of our project those factors could easily be assumed constant, and therefore irrelevant to the relative ranking of the various sites. The spatial and attribute data pertaining to Soil Depth, Rock Permeability and Digital Elevation Model (DEM) were fed into the Arc/Info GIS package and the aspect and slope data were derived as secondary data using the raster 
analysis facility of Arc/Info.

Slope has been classified into the following four classes based on the degree to which it influences the risk of soil erosion.

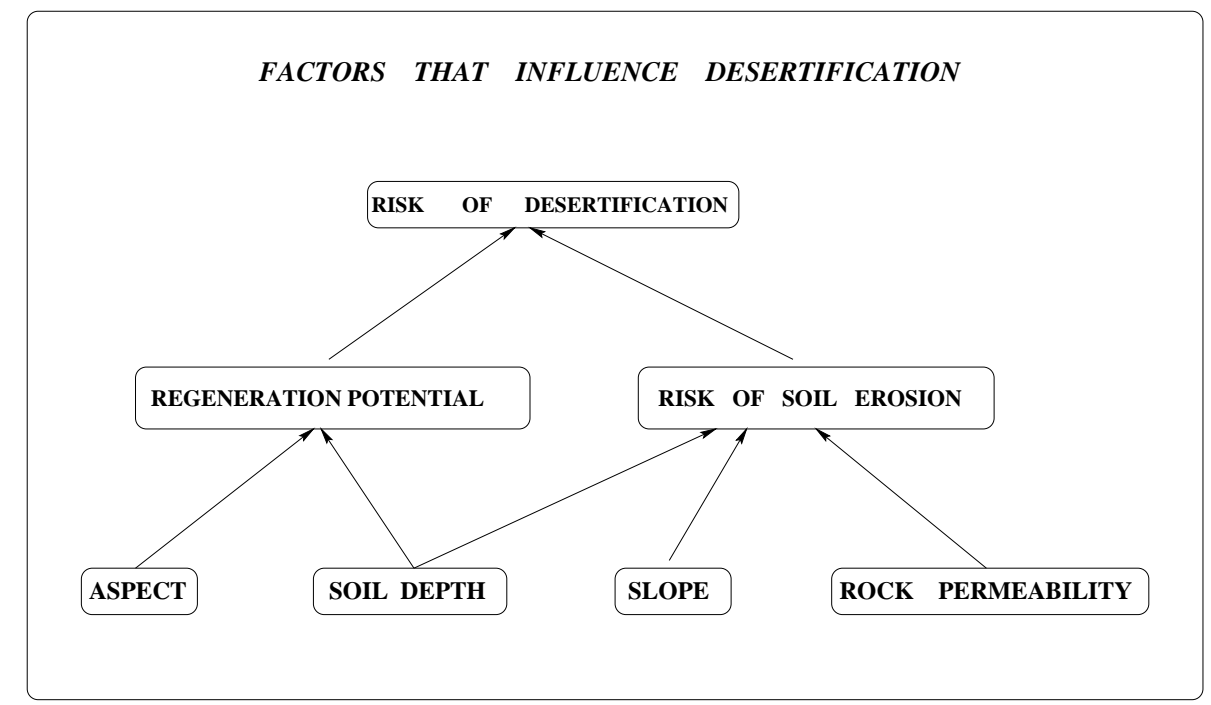

Figure 1: Factors that influence the risk of desertification of a burned forest

1. Gentle: $0-20 \%$

2. Moderate: $21-40 \%$

3. Steep: $>40 \%$

Slope can be expressed in degrees or percentage. When expressed as a percentage the slope is $100 \%$ when the angle is $45^{\circ}$ and approaches $\infty$ as the angle approaches the vertical which is $90^{\circ}$.

Soil Depth has been classified into the following 3 classes.

1. Bare: $<5 \mathrm{~cm}$

2. Shallow: $5-30 \mathrm{~cm}$

3. Deep: $>30 \mathrm{~cm}$

Aspect or orientation of a ridge can be expressed as the angle the normal to the ridge forms with the north direction. This angle could take a value from $0^{\circ}$ to $360^{\circ}$ and it could belong to any of the following classes.

1. North: $0-45^{\circ}, 315-360^{\circ}$ 


\section{East: $45-135^{\circ}$}

3. South: $135-225^{\circ}$

4. West: $225-315^{\circ}$

Rock Permeability refers to the ease with which water may run through the rock. The higher the rock permeability, the lower the risk of soil erosion is. The different types of rock found in the study area are Hard Limestone, Schists, Metamorphic, Calcareous tertiary deposits, Siliceous tertiary deposits and Colluvium. However, the classes used in this study were just 'permeable' and 'impermeable'.

The Arc/Info GIS package was used for performing a few intermediate spatial functions with the data, before any reasoning could be done. While the data regarding Rock Permeability, Soil Depth and DEM were provided for the entire study area, we were concerned with only a few sample sites. Hence, the required data for these sites were extracted by clipping with the sample site boundaries. Slope and aspect values were obtained from the DEM using the GRID module of Arc/Info. GRID is a rule based geo-processing system integrated with Arc/Info.

The reasoning part of the problem under consideration involved the implementation of the hard rules provided by the expert in terms of fuzzy concepts. The rules as given by the expert are shown in Table-I .

\section{RULES FOR NATURAL REGENERATION POTENTIAL}

\begin{tabular}{l|c|ccc|}
\multicolumn{1}{c|}{} & \multicolumn{3}{c}{ SOIL DEPTH } \\
\cline { 3 - 5 } \multicolumn{1}{c|}{} & BARE & SHALLOW & DEEP \\
\hline A & NORTH & SG & SL & NL \\
S & EAST & SG & SL & NL \\
P & & & & \\
E & WEST & SE & ML & SL \\
C & & & & \\
T & SOUTH & SE & ML & SL \\
\hline
\end{tabular}

NL - No Limitation

SL - Slight Limitation

ML - Moderate Limitation

SG - Strong Limitation

SE - Severe limitation 


\begin{tabular}{|c|c|c|c|c|c|c|c|}
\hline & \multicolumn{6}{|c|}{ RULES FOR RISK OF SOIL EROSION } \\
\hline & & \multicolumn{3}{|c|}{ PERMEABLE } & \multicolumn{3}{|c|}{ IMPERMEABLE } \\
\hline & & BARE & SHALLOW & DEEP & BARE & SHALLOW & DEEP \\
\hline $\mathrm{S}$ & GENTLE & * & SR & NSR & * & HR & SR \\
\hline $\mathrm{L}$ & & & & & & & \\
\hline $\mathrm{O}$ & MEDIUM & * & MR & SR & * & VHR & MR \\
\hline $\mathrm{P}$ & & & & & & & \\
\hline $\mathrm{E}$ & STEEP & * & MR & SR & * & VHR & HR \\
\hline
\end{tabular}

* The land with bare soil is already eroded. No further erosion can occur. NSR - No to slight risk

SR - Slight risk

MR - Moderate risk

HR - High risk

VHR - Very high risk

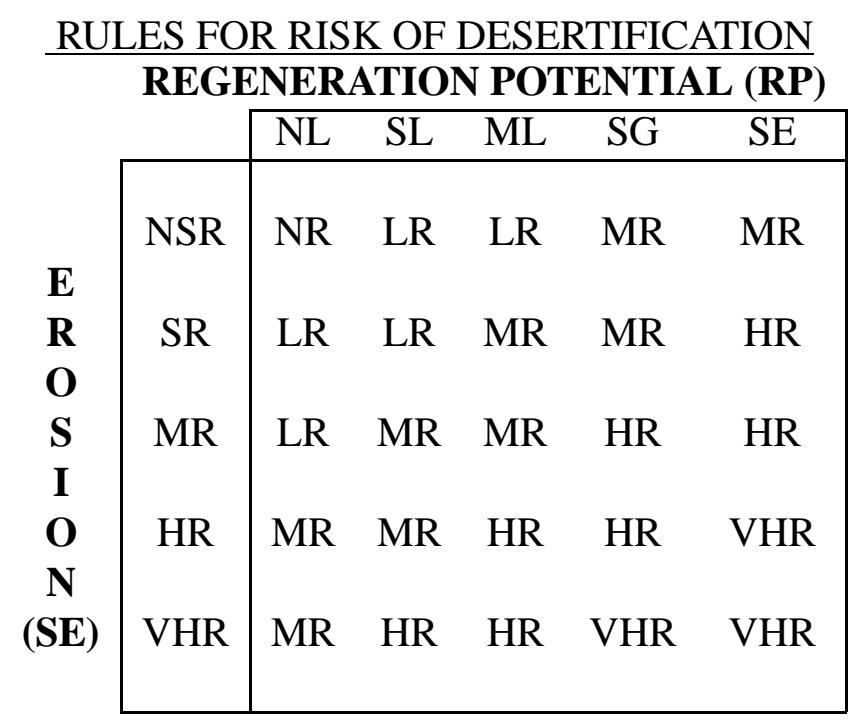

NR - No risk

LR - Low risk

MR - Moderate risk

HR - High risk

VHR - Very high risk 


\section{An overview of Fuzzy Techniques}

Since Zadeh defined fuzzy concepts in 1965 [40], fuzzy logic has been established as the ideal method of dealing with various kinds of uncertainty and vagueness like the ones stated below.

- Experts often express their knowledge in terms of linguistic variables like shallow, medium, deep etc.

- A variable is often characterized by a measurement that takes continuous values. Forcing this variable into one of two or three classes according to the value of its measurement, is too gross and ignores the fact that the transition from one class to the other may be gradual and the boundaries between classes fuzzy.

- There is uncertainty in the measurement of variables especially when many pixel values have to be aggregated together to yield a representative figure.

- There is a mixture of classes within each individual site that is to be classified.

In the problem in question, the fact that each individual site consists of a mixture of classes has been the major motivation for using fuzzy reasoning techniques. Hence, the fuzzy membership values to any particular class of a variable, have been evaluated as the proportion of pixels falling in that class within a particular sample site.

Aggregation operations on fuzzy sets are operations by which several fuzzy sets are combined in a desirable way to produce a single fuzzy set. An aggregation operation on $n$ fuzzy sets where $n \geq 2$ is formally defined by a function

$f:[0,1]^{n} \longrightarrow[0,1]$

When applied to fuzzy sets, this function produces an aggregate fuzzy set by operating on the membership grades of these sets [20]. The nature of aggregation of two variables say $\mathrm{x}$ and $\mathrm{y}$, could be any of the following [5].

1. Aggregation is conjunctive if

$f(x, y) \leq \min (x, y)$

which states that a conjunctive operator has confidence at most as high as the smallest membership value and looks for a simultaneous satisfaction of all criteria that are being combined.

2. Aggregation is disjunctive if

$f(x, y) \geq \max (x, y)$

which states that a disjunctive operator has confidence at least as small as the greatest membership value and looks for a redundancy between the criteria that are being combined.

3. Aggregation is a compromise if $\min (x, y) \leq f(x, y) \leq \max (x, y)$ which is a cautious behaviour. 
The aggregation operators themselves fall under 4 classes, namely

- T-norms

- T-conorms

- Means

- Symmetric Sums

Functions like T-norms and T-conorms have been extensively studied in the literature [5] [2] [20]. The most commonly used T-norms and T-conorms are

Standard Intersection: min

Algebraic Product: $x y$

Bounded Difference: $\max (0, x+y-1)$
Standard Union: $\max$

Probabilistic Sum: $x+y-x y$

Bounded Sum: $\min (1, x+y)$

Min is the smallest T-norm and Max is the largest T-conorm and hence Min is the weakest fuzzy intersection and $\max$ is the strongest fuzzy union. T-norms are conjunctive operators and T-conorms are disjunctive operators.

Apart from these union and intersection operators, there are also other aggregation operators like symmetrical sums and mean operators which are found to be more suitable when we have to deal with aggregation of fuzzy variables refering to different concepts [28] [13] which is also the case in the problem under consideration. By different concepts, we mean aggregation of completely different variables of different scales and nature.

Symmetric Sums take the general form

$$
f(x, y)=\frac{g(x, y)}{g(x, y)+g(1-x, 1-y)}
$$

and their behaviour as to whether they are conjunctive or disjunctive depends on the values of $x$ and $y$. The symmetric sums that have been used in the present study are

$\sigma_{0} \equiv \frac{x y}{1-x-y+2 x y}$ corresponding to $g(x, y)=x y$

$\sigma_{+} \equiv \frac{x+y-x y}{1+x+y-2 x y}$ corresponding to $g(x, y)=x+y-x y$

$\min _{3} \equiv \frac{\min (x, y)}{1-|x-y|}$ corresponding to $g(x, y)=\min (x, y)$

$\max _{3} \equiv \frac{\max (x, y)}{1+|x-y|}$ corresponding to $g(x, y)=\max (x, y)$

where the nature of $\sigma_{0}$ and $\sigma_{+}$depends on the values of $x$ and $y$ while $\min _{3}$ and $\max _{3}$ are compromise operators. Not all symmetric sums are associative.

The most commonly used mean operators are arithmetic mean, harmonic mean and geometric mean and all the mean operators yield a value in between the maximum and minimum and hence have a compromise behaviour. 


\section{Issues of relative importance}

In many applications of fuzzy logic, all variables were considered equally important which may not be true in reality. For example, the two variables Soil Depth and Aspect could affect the Regeneration Potential in different degrees and moreover Soil Depth could influence Regeneration Potential and Soil Erosion also in different degrees. Hence, it may be crucial to include the relative importances of the variables as well in the reasoning process. Yager has studied the issue of inclusion of relative importance in a great detail and has suggested various ways of incorporating it in a multi-criteria decision making problem. In [37], Yager defines an aggregation operator $F: R^{n} \longrightarrow R$ as an ordered weighted aggregation operation of dimension $n$ if it has associated with it a weighting vector $\left(w_{1}, w_{2}, \ldots, w_{n}\right)^{T}$ such that

1. $w_{i} \in[0,1]$,

2. $\sum_{i=1}^{n} w_{i}=1$ and

$F\left(a_{1}, a_{2}, \ldots . a_{n}\right)=\sum_{j=1}^{n} b_{j} w_{j}$ where $b_{j}$ is the jth largest element of $\left(a_{1}, a_{2}, \ldots . . a_{n}\right)$. In [34], Yager evaluated the importance of the objectives in a multi-objective decision making problem by finding the eigenvector of the maximum eigenvalue of a matrix of pairwise comparisons of the importance of each objective. He included the relative importance in the problem by raising each objective to a power representing the respective importance obtained from the eigenvector. When a weight is more than 1 , the higher the weight, the stricter the condition, while when the weight is less than 1 the condition is loosened. Hence, large membership values would be reduced to much smaller than the small ones if their weights are greater than 1, while small membership values will become larger when their weights are smaller than 1 . This ensures that the membership values of less important classes are reduced more, thereby reducing the likelihood of the decision being dominated by those classes, or the membership of more important classes is increased so that the decision is dominated by these classes. In [35], [36] and [34], Yager suggests two other methods of including importances. In each of these, he suggests different ways of doing it, based on whether the nature of fusion is a conjunction or a disjunction. In [35] and [36], Yager states that, a conjunction operation could be performed as

$$
\min \left[I\left(\alpha_{i}, C_{i}\right)\right], i=1,2, \ldots, n
$$

where $\min$ is used as the conjunction operator, $\alpha_{i}$ is the importance of criterion $i$ and $C_{i}$ is the degree of satisfaction of criterion $i$. $I$ indicates the function relating importance and satisfaction and is defined as

Similarly for a disjunction, he suggests

$$
I(a, b) \equiv \max [(1-a), b]
$$

$$
\max \left[U\left(\alpha_{i}, C_{i}\right)\right], i=1,2, \ldots, n
$$

where $\max$ is used as the disjunction operator and

$$
U(a, b) \equiv \min (a, b)
$$

In [34], Yager generalises the method of inclusion of importances. He states that an 'and' operation could be performed as $A\left[I\left(\alpha_{i}, C_{i}\right)\right]$ where $A$ stands for any T-norm operator and $I$ stands for any T-conorm operator. Similarly, for an 'or' operation, he suggests $O\left[U\left(\alpha_{i}, C_{i}\right)\right]$, where $O$ stands for any T-conorm operator and $U$ stands for any T-norm operator. 
In this paper, we explore a new way of incorporating relative importance of the variables: the membership values of two variables say $x$ and $y$ vary between 0 and $w_{1}$ and 0 and $w_{2}$ respectively rather than 0 and 1 . Note that this is not the same as using multiplicative weights for the membership functions, as most of the aggregation operators used are non-linear in $x$ and $y$. Hence most of the operators had to be modified appropriately in order to handle the incorporation of weights. Therefore, the definition of the aggregation function given by $f:[0,1]^{n} \longrightarrow[0,1]$ has been extended to $f:\left[0, w_{1}\right] \cdot\left[0, w_{2}\right] \ldots\left[0, w_{n}\right] \longrightarrow\left[0, \max \left(w_{1}, w_{2}, \ldots w_{n}\right)\right]$ in order to accomodate the weights. The operators as they are originally defined and after the incorporation of weights are shown in Table - II.

For comparison, we also follow Yager and associate weights as powers of the membership values. When this method of weighting is used, the membership values vary between 0 and 1 only and $f:[0,1]^{n} \longrightarrow[0,1]$ and hence there was no necessity to modify the aggregation operators.

Since no quantitative information on the relative importance of the aggregates is available, in both cases, different weight combinations were experimented with. The operators were first used on a training set comprising of 39 sites and the combination of weights which gave the best results when compared with the expert's classification was used in the evaluation with the 14 test sites. 


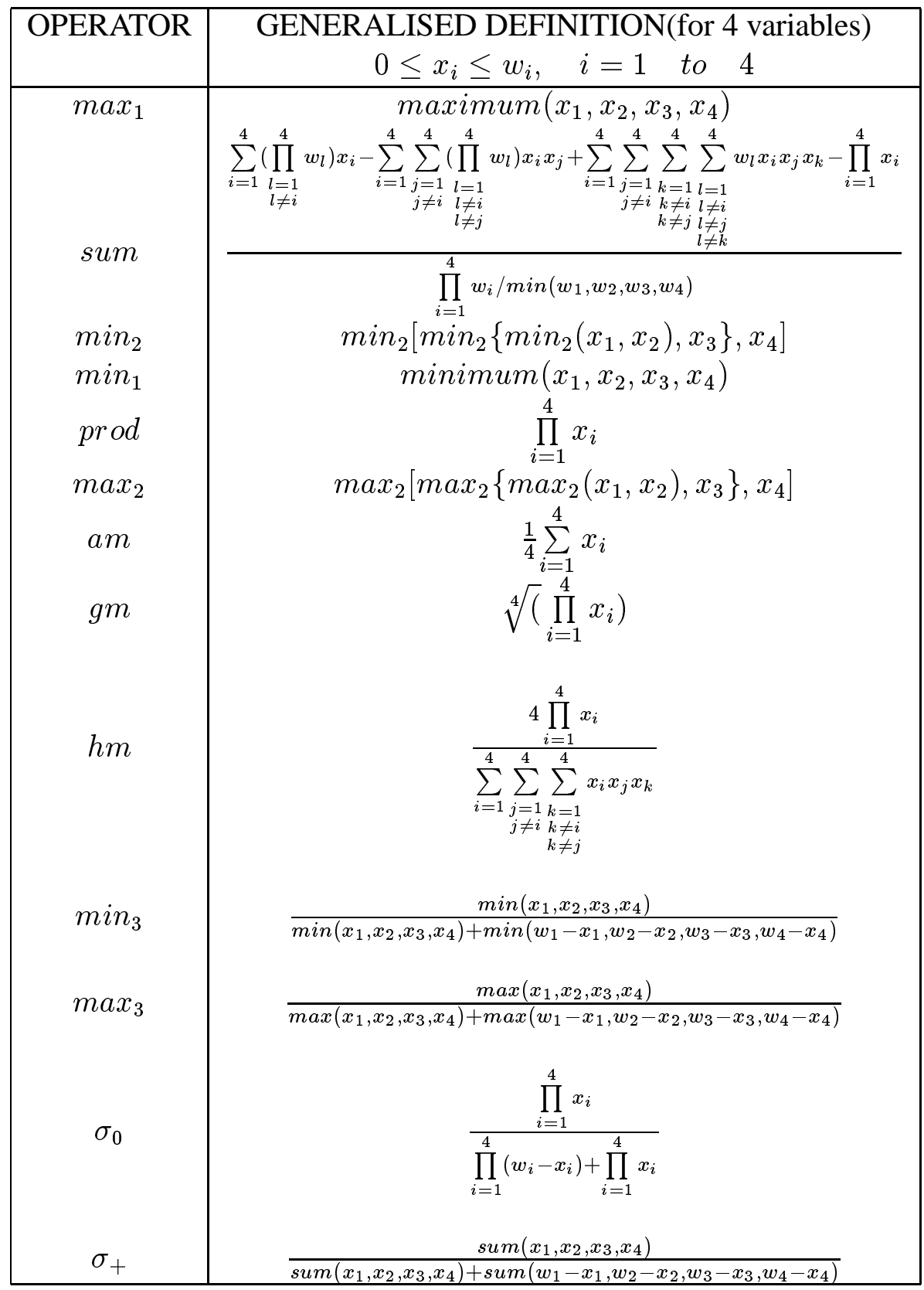

Table 2: Generalised Definition of operators

\section{Application}

It is obvious from table 1, that there are two levels of aggregation of variables, namely and and or and hence two operators have to be combined in various ways to create the composite operator needed: one operator to be used for combining the membership values to the various classes of the independent variables, say for example, Soil Depth and Aspect, and one operator to be used for combining the confidences of the various rules 
that lead to the same classification of the dependent variable, say for example Regeneration Potential. The nature of fusion of information in these two levels is disjunctive and conjunctive respectively i.e., the fusion of information is a conjunction when we combine conditions for certain situations to arise and it is a disjunction when we combine evidence from different rules to lead to the same conclusion. This is schematically represented in figure 2. When the variables involved are fuzzy, these two levels of aggregation could be dealt with fuzzy union and intersection operators.

In this paper, a comparison of the behaviour of all types of aggregation operator is performed. The operators with a conjunctive (disjunctive) nature are applied in the conjunctive (disjunctive) level of the expert rules, and the symmetrical sums and the mean operators are used in both levels. Thus, we have 10 operators each for the conjunctive and the disjunctive levels, creating a total of 100 combination operators when we use the power weighting method. When we use the weighting method proposed here we have 11 operators each creating a total of 121 combination operators as the operators sum and prod behave like a compromise operator and a symmetric sum respectively when generalised. Moreover, since some of the rules involve 3 variables, the operators are generalised to take care of that. All these operators are applied to evaluate the Risk of Soil Erosion, Natural Regeneration Potential and Risk of Desertification independently.

This approach presents two options: We may train the system so that for each sub-

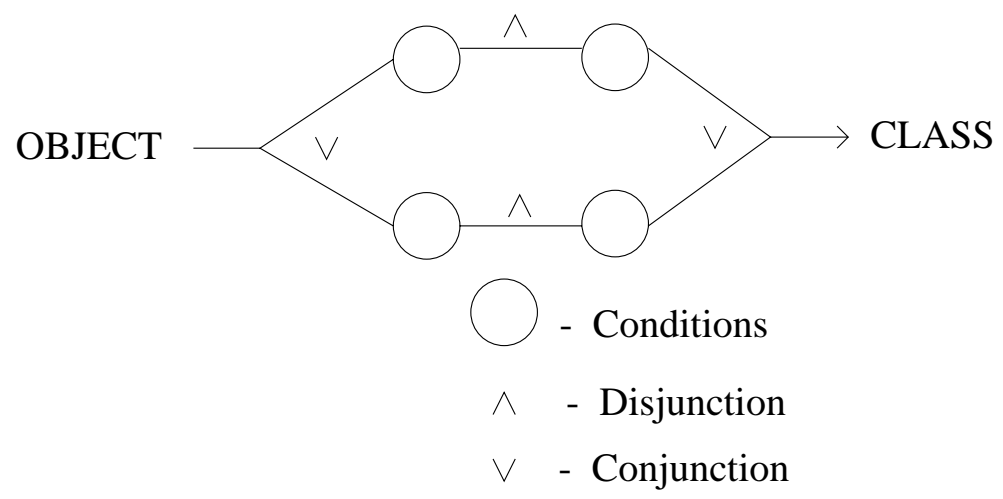

Figure 2: Conjunctive and Disjunctive levels of reasoning

classificaton the best combination of operators is used. This combination could be different for each of the three subproblems. Alternatively, we train the system using for all three subproblems at any one time the same combination of operators for the conjunctive and the disjunctive reasoning. We call these methods Methods I and II respectively. In yet another approach, the rules supplied by the expert may be combined to form rules that directly relate the attributes of a site with the risk of desertification, omitting the intermediate assessment of risk of erosion and regeneration potential. These composite rules are given in table 3 . We call this method Method III.

Each of the three methods was applied with weights used either as membership function powers or as non-unit maximal values of the membership functions, as proposed here. In every case, the weight of one of the variables was fixed to be 1 and different combinations of weights ranging from 0.1 to 10 were experimented with, with the rest of the variables. The operators and the weights that gave the best results in each case with the 39 training 
sites when compared with the expert's classification were then used on the 14 test sites.

\begin{tabular}{|cccc|c|}
\hline Soil depth & Aspect & Rock Permeability & Slope & ROD \\
\hline Deep & North & Permeable & Gentle & NR \\
Deep & East & Permeable & Gentle & NR \\
Deep & South & Permeable & Medium & LR \\
Deep & West & Permeable & Medium & LR \\
Deep & South & Permeable & Steep & LR \\
Deep & West & Permeable & Steep & LR \\
Deep & South & Impermeable & Gentle & LR \\
Deep & West & Impermeable & Gentle & LR \\
Shallow & South & Permeable & Medium & MR \\
Shallow & West & Permeable & Medium & MR \\
Shallow & South & Permeable & Steep & MR \\
Shallow & West & Permeable & Steep & MR \\
Deep & South & Impermeable & Steep & MR \\
Deep & West & Impermeable & Steep & MR \\
Shallow & South & Impermeable & Gentle & HR \\
Shallow & West & Impermeable & Gentle & HR \\
Shallow & South & Impermeable & Medium & VHR \\
Shallow & West & Impermeable & Medium & VHR \\
Shallow & South & Impermeable & Steep & VHR \\
Shallow & West & Impermeable & Steep & VHR \\
\hline
\end{tabular}

ROD - Risk of Desertification

NR - No risk

LR - Low risk

MR - Moderate risk

HR - High risk

VHR - Very high risk

Table 3: Combined rules for Risk Of Desertification

\subsection{Method I:}

The results of method I are presented in tables 4, 5 and 6. The operators and the weights that gave the best results with the training sites were used for the testing sites with no further adjustment. The first column of each set of results gives the number of sites that end up with exactly the same classification as that obtained by the expert, while in the second column we also include sites which were \pm 1 class (out of five possible classes) off from the expert's classification.

In table 4 we list the combination of disjunctive and conjunctive operators and the cor- 
responding weights that produced the best results for the Natural Regeneration Potential. It is interesting to note that both weighting approaches placed higher significance to Soil Depth than to Aspect.

\begin{tabular}{|c|c|c|c|c|c|c|c|c|}
\hline \multirow{2}{*}{$\begin{array}{l}\text { WEIGHTING } \\
\text { PROCEDURE }\end{array}$} & \multicolumn{2}{|c|}{ OPERATOR } & \multicolumn{2}{|r|}{ WEIGHTS } & \multicolumn{2}{|c|}{ TRAINING SITES } & \multicolumn{2}{|c|}{ TEST SITES } \\
\hline & DISJUNCTIVE & CONJUNCTIVE & $\mathrm{SD}$ & $\mathrm{AS}$ & $\mathrm{CCS}$ & CCSE & $\mathrm{CCS}$ & CCSE \\
\hline \multirow{4}{*}{$\begin{array}{c}\text { PROPOSED } \\
\text { HERE }\end{array}$} & $\min _{2}$ & sum & 1 & $0.1,0.2,0.4,0.8$ & 21 & 36 & 8 & 14 \\
\hline & sum & sum & 1 & $0.1,0.2,0.4$ & 21 & 36 & 8 & 14 \\
\hline & $\min _{2}$ & $\max _{3}$ & 1 & 10 & 19 & 29 & 8 & 12 \\
\hline & sum & $\max _{3}$ & 1 & $7-10$ & 13 & 27 & 7 & 12 \\
\hline \multirow[t]{2}{*}{ POWER } & sum & $\max _{3}$ & 1 & 2 & 21 & 37 & 7 & 14 \\
\hline & $\min _{2}$ & $\max _{3}$ & 1 & 2 & 21 & 37 & 7 & 14 \\
\hline
\end{tabular}

SD - Soil Depth, AS - Aspect, CCS - Correctly Classified Sites, CCSE - Correctly Classified Sites with \pm 1 class error

Table 4: Method I: Results for Limitation to Natural Regeneration Potential

For the risk of soil erosion (table 5), the weighting method we propose gave the best results with the operators $\max _{3}$ and sum for the disjunctive and the conjunctive level respectively, while with power weighting the best results were obtained with $\max _{1}$ and $\max _{3}$ operators. For comparison, we also include in the table the results obtained when the combination of operators that worked best for one of the weighting methods was used with the other weighting method. However, while the operator sum behaved as the best conjunctive operator in our weighting method, it is not used in the power weighting method for comparison because the standard sum operator is only a disjunctive operator. We notice that the weighting method proposed here gave slightly better results with the test sites, even though the power method gave slightly better results with the training sites. Relative importances given by the two methods are not consistent. Soil depth appears to be the least important in the weighting method proposed here, while it appears as the most important in the power method.

The results obtained by using the best aggregation operators and weights for Natural Regeneration Potential and Soil Erosion were then used to evaluate the ROD (Risk of Desertification) and the results are given in table 6 . The best set of operators for our weighting method was $\min _{2} \sigma_{+}$with Regeneration Potential considered 5 times more important than Risk of Soil Erosion, while the best set of operators for the power weighting method was $\max _{1} \max _{3}$ with Risk of Soil Erosion considered more important than Regeneration Potential. For comparison, we also present in table 6 the results obtained when we exchanged the set of best operators between the two weighting methods. The results show that there is not a significant difference between the results obtained by our weighting 


\begin{tabular}{|c|c|c|c|c|c|c|c|c|c|}
\hline \multirow{2}{*}{$\begin{array}{l}\text { WEIGHTING } \\
\text { PROCEDURE }\end{array}$} & \multicolumn{2}{|c|}{ OPERATOR } & \multicolumn{3}{|c|}{ WEIGHTS } & \multicolumn{2}{|c|}{ TRAINING SITES } & \multicolumn{2}{|c|}{ TEST SITES } \\
\hline & DISJUNCTIVE & CONJUNCTIVE & SD & $\mathrm{Rp}$ & SL & CCS & CCSE & CCS & CCSE \\
\hline PROPOSED & $\max _{3}$ & sum & 1 & 9 & 9 & 27 & 35 & 7 & 8 \\
\hline HERE & $\max _{1}$ & $\max _{3}$ & 1 & $0.9-7$ & 0.1 & 20 & 36 & 3 & 10 \\
\hline POWER & $\max _{1}$ & $\max _{3}$ & 1 & $5-10$ & 4-(Rp-1) & 29 & 39 & 6 & 12 \\
\hline
\end{tabular}

SD - Soil Depth, ASP - Aspect, Rp - Rock Permeability, SL - Slope, CCS - Correctly Classified Sites, CCSE - Correctly Classified Sites with \pm 1 class error

Table 5: Method I: Results for Risk of Soil Erosion

method and the power method. 
WEIGHTING PROPOSED HERE

\begin{tabular}{|lc|cc|cc|cc|cc|cc|}
\hline \multicolumn{4}{|c|}{ ROD } & \multicolumn{2}{|c|}{ NRP } & \multicolumn{2}{c|}{ SE } & \multicolumn{2}{c|}{ TRAINING } & \multicolumn{2}{c|}{ TEST } \\
\hline DISJ & CONJ & \multicolumn{2}{|c|}{ WEIGHTS } & DISJ & CONJ & DISJ & CONJ & CCS & CCSE & CCS & CCSE \\
& & NRP & SE & & & & & & & & \\
\hline \multirow{2}{*}{$\min _{2}$} & $\sigma_{+}$ & 1 & 0.2 & $\min _{2}$ & sum & $\max _{3}$ & sum & 26 & 33 & 7 & 14 \\
$\max _{1}$ & $\min _{1}$ & 1 & 0.2 & $\min _{2}$ & $\max _{3}$ & $\max$ & $\max _{3}$ & 23 & 33 & 5 & 10 \\
\hline
\end{tabular}

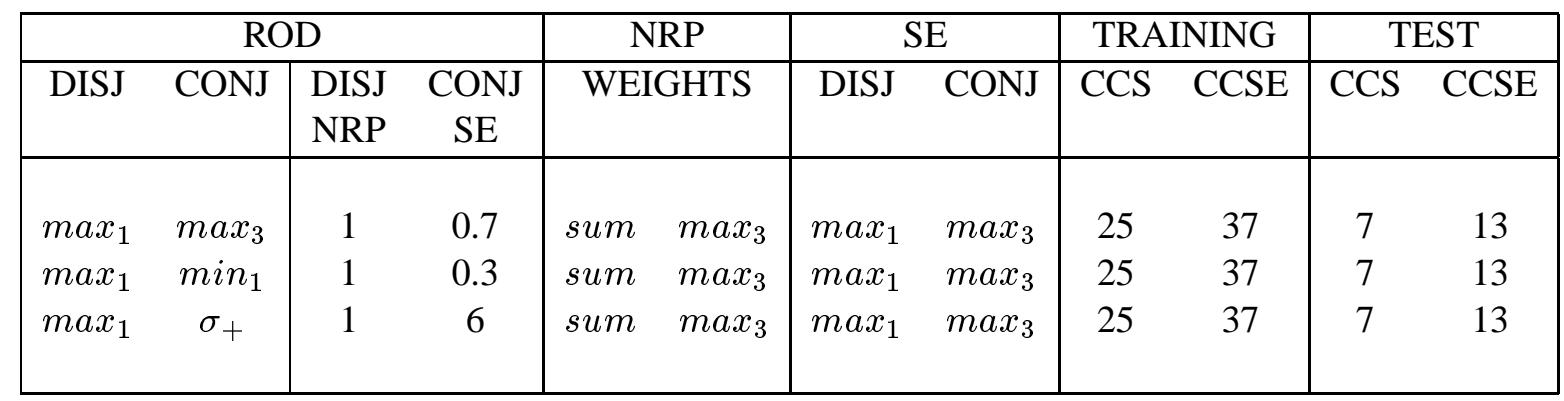

SD - Soil Depth, Rp - Rock Permeability, SL - Slope, CCS - Correctly Classified Sites, CCSE Correctly Classified Sites with 1 class error, ROD - Risk of Desertification, NRP - Natural Regeneration Potential, SE - Risk of Soil Erosion

Table 6: Method I: Results for Risk of Desertification

\subsection{Method II:}

In this method, the same set of aggregation operators was used at all levels of conjunctive/disjunctive reasoning everytime. The best results obtained are given in table 7 . The results obtained are much better than those obtained with Method I. It can be noticed that our weighting approach gave better results than the power method by correctly classifying 9 out of the 14 test sites. Guided from the performance with the training data, one would choose $\mathrm{min}_{2} a \mathrm{~m}$ combination of operators for our weighting approach and the $\min _{2} \max _{3}$ combination for the power approach and achieve 9 and 6 correctly classified sites out of the 14 test sites respectively. The combination $\min _{2} \max _{3}$ that performed the best with power method gave better results with our weighting method. In this case, it is not possible to draw any conclusions about the relative importance of the various factors, as the reasoning is performed in two levels that involve non-linear processing. Note that the Soil Depth attribute was incorporated with separate weights when entered through the Regeneration Potential and Soil Erosion.

\subsection{Method III:}

In this method, instead of evaluating the Risk of Desertification from Regeneration Potential and Soil Erosion, the rules of Regeneration Potential and Soil Erosion were combined to evaluate the Risk of Desertification directly from the fuzzy memberships of the four 
WEIGHTING METHOD PROPOSED HERE

\begin{tabular}{|c|c|c|c|c|c|c|c|c|c|c|c|c|}
\hline \multicolumn{2}{|c|}{ OPERATOR } & \multicolumn{7}{|c|}{ WEIGHTS } & \multicolumn{2}{|c|}{ TRAINING SITES } & \multicolumn{2}{|c|}{ TEST SITES } \\
\hline DISJ. & CONJ. & $S D_{1}$ & ASP & $\mathrm{Rp}$ & SL & $S D_{2}$ & NRP & SE & $\mathrm{CCS}$ & CCSE & $\mathrm{CCS}$ & CCSE \\
\hline $\min _{2}$ & $a m$ & 1 & 0.3 & 0.1 & 1 & 0.5 & 1 & 1 & 37 & 39 & 9 & 12 \\
\hline $\min _{2}$ & $a m$ & 1 & 0.3 & 0.1 & 1 & 0.6 & 1 & 10 & 38 & 39 & 9 & 13 \\
\hline $\min _{2}$ & $\max _{3}$ & 1 & 0.1 & 3 & 1 & 0.1 & 1 & 10 & 32 & 39 & 8 & 12 \\
\hline
\end{tabular}

POWER WEIGHTING

\begin{tabular}{|c|c|c|c|c|c|c|c|c|c|c|c|c|}
\hline \multicolumn{2}{|c|}{ OPERATOR } & \multicolumn{7}{|c|}{ WEIGHTS } & \multicolumn{2}{|c|}{ TRAINING SITES } & \multicolumn{2}{|c|}{ TEST SITES } \\
\hline$\overline{\text { DISJ. }}$ & CONJ. & $\overline{S D_{1}}$ & ASP & $\mathrm{Rp}$ & $\mathrm{SL}$ & $\mathrm{SD}_{2}$ & $\overline{\mathrm{NRP}}$ & $\mathrm{SE}$ & $\overline{\mathrm{CCS}}$ & CCSE & $\mathrm{CCS}$ & $\overline{\mathrm{CCSE}}$ \\
\hline $\min _{2}$ & $\max _{3}$ & 1 & 2 & 6 & 1 & 4 & 1 & 1 & 35 & 38 & 6 & 13 \\
\hline $\min _{2}$ & $a m$ & 1 & 0.5 & 0.5 & 1 & 6 & 1 & 10 & 33 & 39 & 5 & 14 \\
\hline
\end{tabular}

$S D_{1}$ - Soil Depth as a factor of NRP, ASP - Aspect, $S D_{2}$ - Soil Depth as a factor of SE, Rp Rock Permeability, SL - Slope, NRP - Limitation to Natural Regeneration Potential, SE - Risk of Soil Erosion, CCS - Correctly Classified Sites, CCSE - Correctly Classified Sites with 1 class error

Table 7: Method II: Results for Risk of Desertification

basic variables, according to the rules shown in table 3 . The results are given in table 8 . The generalised aggregation operators for four variables presented in table 2 were used in this case. From table 8, it can be seen that the maximum number of correctly classified sites obtained in the training phase is as low as 22 sites out of 39, with our weighting approach and 24 sites with the power approach. This method did not produce good results with the test sites either. Further, the generalisation of the system is not very good. There is also some inconsistency in the ranking of the various factors in order of decreasing importance: Our weighting method gives highest importance to Rock Permeability followed by equal importance to Soil Depth and Aspect followed by Slope. The power weighting gives maximum importance to Slope.

\section{Discussion}

It can be observed that of the above three methods, Method II gave the maximum number of correctly classified sites, when compared with the expert's opinion, but with not very good generalization capabilities. The weighting method proposed here gave marginally better results than the power weighting method. By far, the worst properties were exhibited by Method III. From the fact that Method III did not perform well, we conclude 


\begin{tabular}{|c|cc|ccccc|cc|cc|}
\hline WEIGHTING & \multicolumn{2}{|c|}{ OPERATOR } & \multicolumn{4}{|c|}{ WEIGHTS } & \multicolumn{2}{|c|}{ TRAINING SITES } & \multicolumn{2}{c|}{ TEST SITES } \\
\cline { 2 - 11 } PROCEDURE & DISJ & CONJ & SD & ASP & Rp & SL & CCS & CCSE & CCS & CCSE \\
\hline \multirow{3}{*}{$\begin{array}{c}\text { PROPOSED } \\
\text { HERE }\end{array}$} & sum & am & 1 & 1 & 4 & 0.1 & 22 & 25 & 3 & 8 \\
& min $_{2}$ & $\sigma_{+}$ & 1 & 1 & 1 & 0.1 & 17 & 34 & 4 & 10 \\
& & & & & & & & & & \\
\hline POWER & min $_{2}$ & $\sigma_{+}$ & 3 & 1 & 2 & 0.1 & 24 & 31 & 4 & 8 \\
& sum & am & 0.1 & 1 & 0.1 & 0.1 & 13 & 28 & 0 & 6 \\
& & & & & & & & & & \\
\hline
\end{tabular}

SD - Soil Depth, ASP - Aspect, Rp - Rock Permeability, SL - Slope, CCS - Correctly Classified Sites, CCSE - Correctly Classified Sites with 1 class error

* - many combinations of weights give the same result

Table 8: Method III: Results for Risk of Desertification

that, at least the expert who evaluated the data we used, used the intermediate steps of evaluating Regeneration Potential and Soil Erosion in his reasoning on the problem, and the non-linearities of these two steps of reasoning cannot be modelled satisfactorily with the type of nonlinearity we introduce with our operators and the weighting process.

The importance of using weighting is assessed by presenting in table 9 the results obtained by all three methods when all factors are given equal weight (i.e., by using the classical fuzzy logic approach). The results presented in the table are the best from each method over all possible combinations of operators. It can be seen that the best result achieved with equal weights was by using Method I, that classified correctly 23 out of the 39 training sites and 5 out of the 14 test sites. These results have to be compared with the weighted Method II results: A total of 37 out of 39 training sites were correctly classified and all 39 sites were correctly classified if we allow an error of 1 class deviation, by method II and by the weighting procedure proposed in this paper. For the testing sites one could achieve the correct classification of 9 out of the 14 sites. This proves that the use of weighting greatly improves the performance of the approach.

Since Method II seems to be so much better than all others, we investigated it in more detail. In all the work presented here, memberships are calculated as fractional class components of composite sites. For example, if a site consists of 150 pixels, 30 of which have slope attribute in class 'steep' and 120 have slope attribute 'medium', the site is given $20 \%$ membership to class 'steep' and $80 \%$ to class 'medium'. It has been explained elsewhere [27], how the membership functions can be calculated as integrals of the Gaussianly distributed errors in the measurements. The mean and variance of each Gaussian is estimated from the (numerical) attributes of each region. We used this type of membership function in combination with all possible operators and both ways of weighting, for Method II. We present the results obtained in detail in tables 10 and 11 for the two weighting approaches respectively. The numbers in brackets are the results obtained with membership functions calculated as described in [27]. In general these membership func- 
tions improved the results without altering the conclusion as to which operators are best. With the proposed weighting with $\mathrm{min}_{2}$ am operators, 38 (instead of 37) out of the 39 training sites were correctly classified and 11 (instead of 9) out of the 14 test sites. With the power weighting, the best achieved was with $\min _{2} \max _{3}$ operators with 37 (instead of 35 ) training sites correctly classified and 7 (instead of 6 ) testing sites.

It can also be seen from table 10 that all compromise operators perform quite badly both as disjunctive and conjunctive operators, except $a m$, a compromise operator which gives good results when used as a conjunctive operator. Though am as conjunctive operator performs reasonably well combined with most of the other operators, it gives the best results when combined with $\min _{2}$ as the disjunctive operator. Symmetrical sums do not seem to perform well in either level. Though some of the combinations of disjunctive and conjunctive operators perform reasonably, none of them performs as well as $\min _{2} a m$ i.e., with $\min _{2}$, in the disjunctive level and $a m$ in the conjunctive level. When the weights are used as power of membership values, $\max _{3}$ performs well as a conjunctive operator, though it gives the best results when combined with $\min _{2}$. If we allow one class error, then $\min _{2} a m, \min _{2} \sigma_{+}$and $\min _{2} \max _{3}$ correctly classify all the sites in both cases. Though the same disjunctive operator $\min _{2}$ works well in the disjunctive level of both cases, two different compromise operators perform well in the conjunctive level. 


\begin{tabular}{|c|c|c|c|c|c|c|c|}
\hline \multirow[t]{2}{*}{ METHOD } & \multirow[t]{2}{*}{ RISK } & \multicolumn{2}{|c|}{ OPERATOR } & \multicolumn{2}{|c|}{ TRAINING SITES } & \multicolumn{2}{|c|}{ TEST SITES } \\
\hline & & DISJUNCTIVE & CONJUNCTIVE & $\overline{\mathrm{CCS}}$ & CCSE & CCS & CCSE \\
\hline \multirow[t]{10}{*}{ I } & \multirow[t]{4}{*}{ NRP } & $\min _{2}$ & mean & 18 & 27 & 8 & 12 \\
\hline & & $\min _{2}$ & $a m$ & 18 & 27 & 8 & 12 \\
\hline & & $\min _{2}$ & $\sigma_{+}$ & 18 & 27 & 8 & 12 \\
\hline & & $\min _{2}$ & $\max _{3}$ & 18 & 27 & 8 & 12 \\
\hline & \multirow[t]{4}{*}{ SE } & $g m$ & mean & 20 & 27 & 4 & 5 \\
\hline & & $g m$ & $a m$ & 20 & 27 & 4 & 5 \\
\hline & & $h m$ & mean & 20 & 27 & 4 & 5 \\
\hline & & $h m$ & $a m$ & 20 & 27 & 4 & 5 \\
\hline & ROD & $\min _{2}$ & $g m$ & 23 & 32 & 3 & 12 \\
\hline & & $\min _{2}$ & $\sigma_{+}$ & 23 & 32 & 5 & 14 \\
\hline \multirow[t]{3}{*}{ II } & \multirow[t]{2}{*}{ ROD (prop) } & sum & mean & 17 & 33 & 3 & 10 \\
\hline & & sum & $a m$ & 17 & 33 & 3 & 10 \\
\hline & ROD (gauss) & sum & $\max _{3}$ & 16 & 32 & 3 & 13 \\
\hline \multirow[t]{2}{*}{ III } & \multirow[t]{2}{*}{ ROD } & $\min _{2}$ & $\sigma_{+}$ & 17 & 34 & 4 & 10 \\
\hline & & $\min _{2}$ & $\max _{3}$ & 17 & 34 & 4 & 10 \\
\hline
\end{tabular}

CCS - Correctly Classified Sites, CCSE - Correctly Classified Sites with 1 class error

Table 9: Results with all variables considered equally important 


\section{RESULTS WITH DIFFERENT COMBINATIONS OF AGGREGATION OPERATORS}

- (with proposed weights)

\begin{tabular}{|c|c|c|c|c|c|c|c|c|c|}
\hline \multirow[t]{2}{*}{ OPERS } & \multicolumn{2}{|c|}{ TR } & \multicolumn{2}{|c|}{ TT } & \multirow[t]{2}{*}{ OPERS } & \multicolumn{2}{|c|}{ TR } & \multicolumn{2}{|c|}{ TT } \\
\hline & CCS & CCSE & $\mathrm{CCS}$ & CCSE & & CCS & CCSE & CCS & CCSE \\
\hline $\max _{1}$ sum & $24(27)$ & $33(35)$ & $5(8)$ & $11(14)$ & $h_{m m a x}$ & $9(9)$ & $22(23)$ & $0(1)$ & $3(7)$ \\
\hline $\max _{1} a m$ & $18(12)$ & $30(35)$ & $4(5)$ & $8(8)$ & hmmin $_{3}$ & $0(0)$ & $22(23)$ & $0(0)$ & $1(1)$ \\
\hline $\max _{1} g m$ & $9(8)$ & $22(27)$ & $4(3)$ & $8(7)$ & hmprod & $0(0)$ & $22(23)$ & $0(0)$ & $1(1)$ \\
\hline $\max _{1} h m$ & $9(9)$ & $22(27)$ & $4(3)$ & $8(7)$ & hmmin $_{1}$ & $0(0)$ & $22(23)$ & $0(0)$ & $1(1)$ \\
\hline $\max _{1} \sigma_{0}$ & $10(14)$ & $26(29)$ & $6(5)$ & $8(7)$ & $h_{\operatorname{mmax}}$ & $3(3)$ & $29(28)$ & $1(2)$ & $5(8)$ \\
\hline $\max _{1} \sigma_{+}$ & $15(17)$ & $30(28)$ & $5(4)$ & $11(9)$ & $\sigma_{0} s u m$ & $26(31)$ & $35(38)$ & $3(3)$ & $9(13)$ \\
\hline $\max _{1} \max _{3}$ & $18(18)$ & $32(36)$ & $6(4)$ & $11(11)$ & $\sigma_{0} a m$ & $20(17)$ & $31(35)$ & $2(4)$ & $8(10)$ \\
\hline $\max _{1} \min _{3}$ & $11(14)$ & $26(29)$ & $6(5)$ & $8(7)$ & $\sigma_{0} g m$ & $0(0)$ & $31(35)$ & $0(0)$ & $1(1)$ \\
\hline $\max _{1}$ prod & $9(8)$ & $22(27)$ & $4(3)$ & $8(7)$ & $\sigma_{0} h m$ & $1(1)$ & $3(2)$ & $0(0)$ & $1(1)$ \\
\hline $\max _{1} \min _{1}$ & $12(12)$ & $26(29)$ & $5(4)$ & $9(7)$ & $\sigma_{0} \sigma_{0}$ & $0(0)$ & $3(2)$ & $0(0)$ & $1(1)$ \\
\hline $\max _{1} \max _{2}$ & $17(11)$ & $31(32)$ & $3(3)$ & $8(10)$ & $\sigma_{0} \sigma_{+}$ & 20 (19) & $28(31)$ & $3(2)$ & $6(7)$ \\
\hline min $_{2}$ sum & $32(34)$ & $34(36)$ & $7(8)$ & $13(14)$ & $\sigma_{0} \max _{3}$ & $19(20)$ & $28(33)$ & $2(3)$ & $6(9)$ \\
\hline $\min _{2} a m$ & 37 (38) & 39 (39) & $9(11)$ & $12(14)$ & $\sigma_{0} \min _{3}$ & $1(1)$ & $3(2)$ & $0(0)$ & $1(1)$ \\
\hline $\min _{2} g m$ & $10(11)$ & $25(24)$ & $4(3)$ & $8(6)$ & $\sigma_{0}$ prod & $0(1)$ & $3(2)$ & $0(0)$ & $1(1)$ \\
\hline $\min _{2} h m$ & $10(10)$ & $22(26)$ & $3(2)$ & $7(6)$ & $\sigma_{0} \min$ & $0(0)$ & $3(2)$ & $0(0)$ & $1(1)$ \\
\hline $\min _{2} \sigma_{0}$ & $10(13)$ & $27(29)$ & $6(5)$ & $8(7)$ & $\sigma_{0} \max _{2}$ & $1(1)$ & $6(4)$ & $0(0)$ & $1(1)$ \\
\hline $\min _{2} \sigma_{+}$ & $35(32)$ & 39 (39) & $10(6)$ & $12(14)$ & $\sigma_{+} s u m$ & $20(20)$ & $37(38)$ & $2(2)$ & $11(14)$ \\
\hline $\min _{2} \max _{3}$ & 19 (19) & 39 (39) & $9(8)$ & $14(14)$ & $\sigma_{+} a m$ & $18(19)$ & $32(32)$ & $1(4)$ & $9(9)$ \\
\hline $\min _{2} \min _{3}$ & $11(13)$ & $27(29)$ & $6(5)$ & $8(7)$ & $\sigma_{+} g m$ & $8(9)$ & $24(24)$ & $3(2)$ & 7 ( 6) \\
\hline $\min _{2}$ prod & $10(11)$ & $22(24)$ & $5(3)$ & $7(6)$ & $\sigma_{+} h m$ & $8(9)$ & $23(27)$ & $4(3)$ & $8(7)$ \\
\hline $\min _{2} \min _{1}$ & $11(12)$ & $23(29)$ & $4(3)$ & $9(7)$ & $\sigma_{+} \sigma_{0}$ & 7 ( 9) & $20(25)$ & $4(2)$ & $6(6)$ \\
\hline $\min _{2} \max _{2}$ & $18(17)$ & $32(35)$ & $2(3)$ & 7 (10) & $\sigma_{+} \sigma_{+}$ & $22(18)$ & $30(30)$ & $2(1)$ & 7 ( 8) \\
\hline sumsum & $26(29)$ & 39 (37) & $0(2)$ & $9(12)$ & $\sigma_{+} \max _{3}$ & $18(19)$ & $26(29)$ & $0(2)$ & $6(7)$ \\
\hline sumam & $25(24)$ & $34(33)$ & $4(5)$ & $9(8)$ & $\sigma_{+} \min _{3}$ & $8(10)$ & $21(25)$ & $4(2)$ & $6(6)$ \\
\hline sumgm & $9(9)$ & $23(26)$ & $4(3)$ & $8(6)$ & $\sigma_{+}$prod & $9(9)$ & $23(24)$ & $4(2)$ & $8(6)$ \\
\hline sumhm & $10(9)$ & $22(27)$ & $4(3)$ & $8(7)$ & $\sigma_{+} \min$ & $10(9)$ & $23(27)$ & $3(2)$ & 7 ( 6) \\
\hline sum $\sigma_{0}$ & $10(13)$ & $26(29)$ & $6(5)$ & $8(7)$ & $\sigma_{+} \max _{2}$ & $7(11)$ & $24(27)$ & $2(1)$ & $6(9)$ \\
\hline sum $_{+}$ & $20(18)$ & $32(34)$ & $4(3)$ & $10(10)$ & $\max _{3}$ sum & $15(20)$ & $32(32)$ & $2(2)$ & $10(12)$ \\
\hline summax $_{3}$ & $22(23)$ & $36(37)$ & $5(3)$ & $11(11)$ & $\max _{3} a m$ & $16(17)$ & $29(32)$ & $1(3)$ & 8 (9) \\
\hline summin $_{3}$ & $11(14)$ & $26(29)$ & $6(5)$ & $8(7)$ & $\max _{3} g m$ & $9(8)$ & $22(26)$ & $3(2)$ & $7(7)$ \\
\hline
\end{tabular}

contd $\ldots$

TR - Training Sites

TT - Test Sites

CCS - Correctly Classified Sites

CCSE - Correctly Classified Sites with 1 class error 


\begin{tabular}{|c|c|c|c|c|c|c|c|c|c|}
\hline \multirow[t]{2}{*}{ OPERS } & \multicolumn{2}{|c|}{ TR } & \multicolumn{2}{|c|}{ TT } & \multirow[t]{2}{*}{ OPERS } & \multicolumn{2}{|c|}{$\mathrm{TR}$} & \multicolumn{2}{|c|}{ TT } \\
\hline & CCS & CCSE & CCS & CCSE & & CCS & CCSE & $\mathrm{CCS}$ & CCSE \\
\hline sumprod & $10(9)$ & $22(23)$ & $4(3)$ & $8(7)$ & $\max _{3} h m$ & $10(9)$ & $22(27)$ & $4(3)$ & $8(7)$ \\
\hline summin $_{1}$ & $11(12)$ & $23(29)$ & $4(3)$ & $9(7)$ & $\max _{3} \sigma_{0}$ & 7 ( 9) & $20(24)$ & $4(2)$ & $6(6)$ \\
\hline summax $_{2}$ & $16(18)$ & $27(35)$ & $1(4)$ & $7(11)$ & $\max _{3} \sigma_{+}$ & $16(18)$ & $29(26)$ & $1(1)$ & $7(6)$ \\
\hline amsum & $16(18)$ & $33(31)$ & $2(2)$ & $11(12)$ & $\max _{3} \max _{3}$ & $14(17)$ & $28(32)$ & $0(3)$ & $7(9)$ \\
\hline amam & $21(21)$ & $30(31)$ & $1(2)$ & $5(9)$ & $\max _{3} \min _{3}$ & $8(10)$ & $21(25)$ & $4(2)$ & $6(5)$ \\
\hline amgm & $5(7)$ & $21(24)$ & $3(2)$ & $7(6)$ & $\max _{3}$ prod & $9(8)$ & $22(27)$ & $4(3)$ & $8(7)$ \\
\hline$a m h m$ & $7(9)$ & $21(24)$ & $3(2)$ & $7(6)$ & $\max _{3} \min _{1}$ & $11(10)$ & $22(26)$ & $4(2)$ & 7 ( 6) \\
\hline$a m \sigma_{0}$ & $6(8)$ & $20(25)$ & $3(2)$ & $7(6)$ & $\max _{3} \max _{2}$ & $5(5)$ & $22(22)$ & $2(4)$ & $3(6)$ \\
\hline$a m \sigma_{+}$ & $19(18)$ & $28(27)$ & $1(2)$ & $5(7)$ & $\min _{3}$ sum & $19(22)$ & $37(34)$ & $5(5)$ & $11(8)$ \\
\hline $\operatorname{ammax}_{3}$ & $18(19)$ & $26(30)$ & $1(2)$ & $5(7)$ & $\min _{3} a m$ & $18(15)$ & $29(32)$ & $3(5)$ & $6(9)$ \\
\hline $\operatorname{ammin}_{3}$ & 7 ( 9) & $21(25)$ & $3(2)$ & $7(6)$ & $\min _{3} g m$ & $1(1)$ & $3(2)$ & $0(0)$ & $1(1)$ \\
\hline amprod & $7(8)$ & $21(26)$ & $3(2)$ & $7(6)$ & $\min _{3} h m$ & $1(1)$ & $3(2)$ & $0(0)$ & $1(1)$ \\
\hline $\operatorname{ammin}_{1}$ & $8(9)$ & $21(25)$ & $3(2)$ & $7(6)$ & $\min _{3} \sigma_{0}$ & $0(0)$ & $3(2)$ & $0(0)$ & $1(1)$ \\
\hline $\operatorname{ammax}_{2}$ & $5(3)$ & $23(21)$ & $0(1)$ & $5(6)$ & $\min _{3} \sigma_{+}$ & 17 (19) & $29(35)$ & $1(2)$ & $9(10)$ \\
\hline gmsum & $16(18)$ & $32(31)$ & $2(3)$ & $11(12)$ & $\min _{3} \max _{3}$ & $14(16)$ & $26(33)$ & $0(4)$ & $6(9)$ \\
\hline gmam & $15(18)$ & $23(28)$ & $0(2)$ & $4(8)$ & $\min _{3} \min _{3}$ & $1(1)$ & $3(2)$ & $0(0)$ & $1(1)$ \\
\hline$g m g m$ & $0(0)$ & $23(28)$ & $0(0)$ & $1(1)$ & $\min _{3}$ prod & $1(1)$ & $3(2)$ & $0(0)$ & $1(1)$ \\
\hline gmhm & $0(1)$ & $23(2)$ & $0(0)$ & $1(1)$ & $\min _{3} \min _{1}$ & $1(1)$ & $3(2)$ & $0(0)$ & $1(1)$ \\
\hline$g m \sigma_{0}$ & $0(0)$ & $23(2)$ & $0(0)$ & $1(1)$ & $\min _{3} \max _{2}$ & $0(0)$ & $3(2)$ & $0(0)$ & $0(0)$ \\
\hline$g m \sigma_{+}$ & $18(19)$ & $25(28)$ & $1(2)$ & $5(7)$ & prodsum & $14(13)$ & $32(29)$ & $1(2)$ & $10(7)$ \\
\hline$g_{\operatorname{mmax}}$ & 17 (19) & $23(30)$ & $1(2)$ & $5(7)$ & prodam & $21(23)$ & $30(35)$ & $2(4)$ & $6(8)$ \\
\hline$g_{m m i n}$ & $1(1)$ & $3(2)$ & $0(0)$ & $1(1)$ & prodgm & $0(1)$ & $30(2)$ & $0(0)$ & $1(1)$ \\
\hline gmprod & $0(0)$ & $3(2)$ & $0(0)$ & $1(1)$ & prodhm & $0(1)$ & $30(2)$ & $0(0)$ & $1(1)$ \\
\hline$g_{m m i n}$ & $1(1)$ & $3(2)$ & $0(0)$ & $1(1)$ & $p r o d \sigma_{0}$ & $0(0)$ & $30(2)$ & $0(0)$ & $1(1)$ \\
\hline $\operatorname{gmmax}_{2}$ & $0(1)$ & $3(4)$ & $0(0)$ & $0(1)$ & $\operatorname{prod}_{+}$ & $5(6)$ & 19 (19) & $3(3)$ & $6(7)$ \\
\hline hmsum & $13(13)$ & $31(29)$ & $3(2)$ & $5(7)$ & prodmax $_{3}$ & $0(3)$ & $19(22)$ & $0(2)$ & $4(9)$ \\
\hline hmam & $18(18)$ & 27 (29) & $2(3)$ & $6(7)$ & prodmin $_{3}$ & $0(0)$ & $19(22)$ & $0(0)$ & $1(1)$ \\
\hline hmgm & $0(0)$ & 27 (29) & $0(0)$ & $1(1)$ & prodprod & $0(1)$ & $19(2)$ & $0(0)$ & $1(1)$ \\
\hline$h m h m$ & $0(1)$ & $27(2)$ & $0(0)$ & $1(1)$ & prodmin $_{1}$ & $0(0)$ & 19 ( 2) & $0(0)$ & $1(1)$ \\
\hline$h m \sigma_{0}$ & $0(0)$ & $27(2)$ & $0(0)$ & $1(1)$ & prodmax $_{2}$ & $11(11)$ & $32(32)$ & $3(5)$ & $5(8)$ \\
\hline$h m \sigma_{+}$ & $12(12)$ & $25(23)$ & $1(2)$ & $4(7)$ & & & & & \\
\hline
\end{tabular}

TR - Training Sites

TT - Test Sites

CCS - Correctly Classified Sites

CCSE - Correctly Classified Sites with 1 class error

Table 10: Results for different combinations of aggregation operators (proposed weights) 


\title{
RESULTS WITH DIFFERENT COMBINATIONS OF AGGREGATION OPERATORS
}

\author{
- (with power weights)
}

\begin{tabular}{|c|c|c|c|c|c|c|c|c|c|}
\hline \multirow[t]{2}{*}{ OPERS } & \multicolumn{2}{|c|}{ TR } & \multicolumn{2}{|c|}{ TT } & \multirow[t]{2}{*}{ OPERS } & \multicolumn{2}{|c|}{ TR } & \multicolumn{2}{|c|}{ TT } \\
\hline & CCS & CCSE & CCS & CCSE & & $\mathrm{CCS}$ & CCSE & CCS & CCSE \\
\hline $\max _{1} a m$ & $15(9)$ & $29(37)$ & $4(5)$ & 9 (9) & hmam & $3(9)$ & $16(24)$ & $1(1)$ & $8(7)$ \\
\hline $\max _{1} g m$ & $9(9)$ & $22(27)$ & $4(3)$ & $8(6)$ & hmgm & $0(0)$ & $16(24)$ & $0(0)$ & $1(1)$ \\
\hline $\max _{1} h m$ & $9(9)$ & $22(27)$ & $4(3)$ & $8(6)$ & $h m h m$ & $0(0)$ & $16(24)$ & $0(0)$ & $1(1)$ \\
\hline $\max _{1} \sigma_{0}$ & $12(15)$ & 27 (29) & $5(4)$ & $8(7)$ & $h m \sigma_{0}$ & $1(1)$ & $3(2)$ & $0(0)$ & $1(1)$ \\
\hline $\max _{1} \sigma_{+}$ & $23(25)$ & $33(34)$ & $2(4)$ & $10(12)$ & $h m \sigma_{+}$ & $17(18)$ & $33(29)$ & $3(2)$ & $8(7)$ \\
\hline $\max _{1} \max _{3}$ & $26(26)$ & $37(36)$ & $7(8)$ & $11(10)$ & $h_{\operatorname{mmax}}$ & $3(6)$ & $13(22)$ & $1(1)$ & $7(7)$ \\
\hline $\max _{1} \min _{3}$ & $11(14)$ & $27(29)$ & $3(5)$ & $8(7)$ & hmmin $_{3}$ & $2(2)$ & $4(3)$ & $0(0)$ & $1(1)$ \\
\hline $\max _{1}$ prod & $9(9)$ & $22(27)$ & $4(3)$ & $8(6)$ & hmprod & $0(0)$ & $4(3)$ & $0(0)$ & $1(1)$ \\
\hline $\max _{1} \min _{1}$ & $12(11)$ & $24(29)$ & $4(4)$ & $9(7)$ & hmmin $_{1}$ & $0(0)$ & $4(3)$ & $0(0)$ & $1(1)$ \\
\hline $\max _{1} \max _{2}$ & $17(16)$ & $29(26)$ & $6(5)$ & $8(9)$ & $h_{m m a x}$ & $0(1)$ & $4(3)$ & $0(0)$ & $1(1)$ \\
\hline $\mathrm{min}_{2} a m$ & $12(13)$ & 39 (39) & $7(6)$ & $14(14)$ & $\sigma_{0} a m$ & $11(15)$ & $29(35)$ & $3(4)$ & 7 (10) \\
\hline $\min _{2} g m$ & $10(9)$ & $26(27)$ & $4(3)$ & $8(7)$ & $\sigma_{0} g m$ & $0(1)$ & $29(2)$ & $0(0)$ & $1(1)$ \\
\hline $\min _{2} h m$ & $10(9)$ & $25(27)$ & $4(3)$ & $8(6)$ & $\sigma_{0} h m$ & $0(1)$ & $29(2)$ & $0(0)$ & $1(1)$ \\
\hline $\min _{2} \sigma_{0}$ & $12(14)$ & $26(28)$ & $7(4)$ & $8(7)$ & $\sigma_{0} \sigma_{0}$ & $1(1)$ & $3(2)$ & $0(0)$ & $1(1)$ \\
\hline $\min _{2} \sigma_{+}$ & $33(33)$ & $39(39)$ & $8(12)$ & $11(14)$ & $\sigma_{0} \sigma_{+}$ & $19(16)$ & $28(30)$ & $3(2)$ & $7(8)$ \\
\hline $\min _{2} \max _{3}$ & 35 (37) & 38 (39) & $6(7)$ & $13(14)$ & $\sigma_{0} \max _{3}$ & $19(20)$ & $31(37)$ & $2(4)$ & $9(12)$ \\
\hline $\min _{2} \min _{3}$ & 15 (14) & $28(26)$ & $5(3)$ & $8(7)$ & $\sigma_{0} \min _{3}$ & $2(2)$ & $4(2)$ & $0(0)$ & $1(1)$ \\
\hline $\min _{2}$ prod & $9(9)$ & $24(27)$ & $4(3)$ & $8(6)$ & $\sigma_{0} p r o d$ & $0(0)$ & $4(2)$ & $0(0)$ & $1(1)$ \\
\hline $\min _{2} \min _{1}$ & $12(11)$ & $24(29)$ & $4(3)$ & $9(7)$ & $\sigma_{0} \min _{1}$ & $0(1)$ & $4(2)$ & $0(0)$ & $1(1)$ \\
\hline $\min _{2} \max _{2}$ & $21(12)$ & $29(38)$ & $2(4)$ & 8 (10) & $\sigma_{0} \max _{2}$ & $1(1)$ & $6(3)$ & $0(0)$ & $4(1)$ \\
\hline sumam & $17(15)$ & $33(38)$ & $3(2)$ & $10(13)$ & $\sigma_{+} a m$ & $13(12)$ & $26(27)$ & $1(2)$ & $7(8)$ \\
\hline sumgm & $9(9)$ & $24(27)$ & $4(3)$ & $8(6)$ & $\sigma_{+} g m$ & $8(9)$ & $22(24)$ & $3(2)$ & $7(6)$ \\
\hline sumhm & $9(9)$ & $24(27)$ & $4(3)$ & $8(6)$ & $\sigma_{+} h m$ & $9(9)$ & $22(26)$ & $3(2)$ & $7(6)$ \\
\hline sum $\sigma_{0}$ & $12(14)$ & 27 (29) & $6(5)$ & $9(7)$ & $\sigma_{+} \sigma_{0}$ & $9(12)$ & $22(23)$ & $3(1)$ & $6(5)$ \\
\hline sum $\sigma_{+}$ & $24(23)$ & $29(31)$ & $2(3)$ & 7 (13) & $\sigma_{+} \sigma_{+}$ & $23(22)$ & $36(31)$ & $3(4)$ & $9(9)$ \\
\hline
\end{tabular}

contd ...

TR - Training Sites

TT - Test Sites

CCS - Correctly Classified Sites

CCSE - Correctly Classified Sites with 1 class error 


\begin{tabular}{|c|c|c|c|c|c|c|c|c|c|}
\hline \multirow[t]{2}{*}{ OPERS } & \multicolumn{2}{|c|}{ TR } & \multicolumn{2}{|c|}{ TT } & \multirow[t]{2}{*}{ OPERS } & \multicolumn{2}{|c|}{ TR } & \multicolumn{2}{|c|}{ TT } \\
\hline & CCS & CCSE & CCS & CCSE & & CCS & CCSE & CCS & CCSE \\
\hline $\operatorname{summax}_{3}$ & $26(24)$ & $36(36)$ & $2(3)$ & $10(13)$ & $\sigma_{+} \max _{3}$ & $26(24)$ & $36(35)$ & $2(3)$ & $10(12)$ \\
\hline summin $_{3}$ & $11(13)$ & $22(28)$ & $1(5)$ & $5(6)$ & $\sigma_{+} \min _{3}$ & $11(14)$ & $25(25)$ & $4(4)$ & $7(6)$ \\
\hline sumprod & $9(9)$ & $23(27)$ & $4(3)$ & $8(6)$ & $\sigma_{+}$prod & $10(9)$ & $22(27)$ & $4(3)$ & $7(6)$ \\
\hline summin $_{1}$ & $12(11)$ & $24(29)$ & $4(3)$ & $9(7)$ & $\sigma_{+} \min _{1}$ & $10(10)$ & $22(29)$ & $4(3)$ & $8(7)$ \\
\hline summax $_{2}$ & $16(8)$ & $31(34)$ & $4(3)$ & $7(8)$ & $\sigma_{+} \max _{2}$ & $10(6)$ & $28(29)$ & $3(1)$ & $7(6)$ \\
\hline amam & $12(12)$ & $27(27)$ & $1(3)$ & $7(9)$ & $\max _{3} a m$ & $13(12)$ & $25(27)$ & $1(3)$ & 7 ( 9) \\
\hline amgm & $8(9)$ & $22(24)$ & $3(2)$ & 7 (6) & $\max _{3} g m$ & 8 (9) & $22(24)$ & $3(2)$ & $7(5)$ \\
\hline amhm & $9(9)$ & $22(26)$ & $3(2)$ & $7(5)$ & $\max _{3} h m$ & $9(9)$ & $22(26)$ & $3(2)$ & $7(6)$ \\
\hline$a m \sigma_{0}$ & $9(10)$ & $24(24)$ & $4(2)$ & $7(6)$ & $\max _{3} \sigma_{0}$ & $10(12)$ & $23(25)$ & $4(2)$ & $8(5)$ \\
\hline$a m \sigma_{+}$ & $22(20)$ & $35(32)$ & $0(3)$ & $6(12)$ & $\max _{3} \sigma_{+}$ & $20(20)$ & $27(30)$ & $4(2)$ & $6(8)$ \\
\hline $\operatorname{ammax}_{3}$ & $17(20)$ & $28(30)$ & $2(2)$ & $8(9)$ & $\max _{3} \max _{3}$ & $18(17)$ & $28(32)$ & $1(3)$ & 7 ( 9) \\
\hline $\operatorname{ammin}_{3}$ & $11(12)$ & $22(24)$ & $4(5)$ & $8(5)$ & $\max _{3} \min _{3}$ & $10(10)$ & $20(23)$ & $3(2)$ & $6(4)$ \\
\hline amprod & $10(9)$ & $22(26)$ & $3(2)$ & $7(5)$ & $\max _{3} \operatorname{prod}$ & $10(9)$ & $22(27)$ & $4(3)$ & $7(6)$ \\
\hline $\operatorname{ammin}_{1}$ & $10(9)$ & $22(27)$ & $3(2)$ & $7(5)$ & $\max _{3} \min _{1}$ & $11(10)$ & $22(29)$ & $4(4)$ & $7(7)$ \\
\hline $\operatorname{ammax}_{2}$ & $8(5)$ & $24(25)$ & $2(1)$ & $7(7)$ & $\max _{3} \max _{2}$ & $10(6)$ & $28(30)$ & $3(1)$ & 7 ( 6) \\
\hline gmam & $11(12)$ & $27(26)$ & $0(1)$ & $6(7)$ & $\min _{3} a m$ & $11(14)$ & $24(27)$ & $2(2)$ & $5(9)$ \\
\hline$g m g m$ & $1(1)$ & $3(2)$ & $0(0)$ & $1(1)$ & $\min _{3} g m$ & $1(1)$ & $3(2)$ & $0(0)$ & $1(1)$ \\
\hline gmhm & $1(1)$ & $3(2)$ & $0(0)$ & $1(1)$ & $\min _{3} h m$ & $0(1)$ & $3(2)$ & $0(0)$ & $1(1)$ \\
\hline$g m \sigma_{0}$ & $1(1)$ & $3(2)$ & $0(0)$ & $1(1)$ & $\min _{3} \sigma_{0}$ & $3(2)$ & $6(3)$ & $0(0)$ & $1(1)$ \\
\hline$g m \sigma_{+}$ & 19 (19) & $28(26)$ & $0(1)$ & $6(6)$ & $\min _{3} \sigma_{+}$ & $20(21)$ & 29 (29) & $2(1)$ & $6(6)$ \\
\hline$g_{\operatorname{mmax}}$ & $16(16)$ & $28(32)$ & $2(2)$ & $7(8)$ & $\min _{3} \max _{3}$ & $16(18)$ & $28(35)$ & $2(3)$ & $4(12)$ \\
\hline gmmin $_{3}$ & $2(2)$ & $3(3)$ & $0(0)$ & $1(1)$ & $\min _{3} \min _{3}$ & $5(12)$ & $10(17)$ & $2(1)$ & $5(3)$ \\
\hline gmprod & $1(1)$ & $3(2)$ & $0(0)$ & $1(1)$ & $\min _{3}$ prod & $0(1)$ & $10(2)$ & $0(0)$ & $1(1)$ \\
\hline gmmin $_{1}$ & $1(1)$ & $3(2)$ & $0(0)$ & $1(1)$ & $\min _{3} \min _{1}$ & $1(1)$ & $3(2)$ & $0(0)$ & $1(1)$ \\
\hline$g \max _{2}$ & $0(1)$ & $3(3)$ & $0(0)$ & $0(1)$ & $\min _{3} \max _{2}$ & $0(1)$ & $3(4)$ & $0(0)$ & $0(1)$ \\
\hline
\end{tabular}

TR - Training Sites

TT - Test Sites

CCS - Correctly Classified Sites

CCSE - Correctly Classified Sites with 1 class error

Table 11: Results for different combinations of aggregation operators (power weights)

As a final comparison, we would like to compare all the above discussed results with those that would have been obtained if the fuzzy nature of the problem had been totally ignored. The Arc/Info GIS was used for a simple rule-based reasoning. A Digital Elevation Model (DEM) was used to create the Slope and Apsect layers with the GRID analysis facility of Arc/Info. The layers on Aspect and Soil depth were integrated using the over- 
lay facility and the rules given in table 1 were used to create the output layer reflecting the ranking of 'Limitation to Natural Regeneration Potential'. Similarly, the layers on Soil Depth, Slope and Rock Permeability were integrated and the rules were used to create the layer on 'Risk of Soil Erosion'. The two layers on 'Limitation to Natural Regeneration Potential' and 'Risk of Soil Erosion' were integrated and the rules for Risk of Desertification were used to create the output layer on 'Risk of Desertification'. Since there is no training procedure involved, all 53 sites were lumped together and experimented without distinguishing between the training and the test sites. The comparison showed that only 17 out of 53 sites were correctly classified.

\section{Conclusions}

The results presented in this paper advocate the following points:

1. The use of Fuzzy Logic when reasoning with a GIS, as opposed to a rule-based approach. This conjecture is proved by the fact that when a rule-based approach was used with the GIS data, only 17 out of 53 sites could be correctly classified.

2. The use of integrals of Gaussians as membership functions [27] in preference to the aggregate membership functions calculated as fractions of pixels of each site that belong to a certain class. The former method gave marginally better results than the latter.

3. The use of weights of importance for the various aggregates. This point was proved by comparing the results with those obtained by the classical no-weighting approach.

4. The use of operators other than the conventional min and max operators. The importance of this can be judged by the variety of results obtained with different operators.

The last two points introduce the necessity of a training stage for each problem, when the best operators and the best set of weights can be chosen.

This work was partly supported by a British Council grant and the European grant number EV5V 0025 under the Environment and Climate programme.

\section{References}

[1] J Adams. Wildfire monitoring in New South Wales. International Journal of Remote Sensing, 15(18):3641-3642, 1994.

[2] H Bandemer and S Gottwald. Fuzzy Sets, Fuzzy Logic and Fuzzy Methods with Applications. Wiley, 1995.

[3] $\mathrm{C}$ Banninger and $\mathrm{H}$ Gallaun. A remote sensing approach to monitoring regeneration and predicting risk of erosion and desertification after a forest fire in the Mediterranean region. In Proc. of the Intl. workshop on Remote Sensing and GIS applications to Forest Fire Management, pages 72-74, University of Alcala de Henares, September 1995.

[4] E Binaghi and A Rampini. Fuzzy decision making in the classification of multisource remote sensing data. Optical Engineering, 32(6):1193-1204, 1993. 
[5] I Bloch. Information Combination Operators for Data Fusion: A comparative review with classification. IEEE transactions on System, Man and Cybernetics, 26(1):5267, January 1996.

[6] G F Bonham-Carter, F P Agterberg, and D F Wright. Integration of Geological Datasets for Gold Exploration in Nova Scotia. Photogrammetric Engineering and Remote Sensing, 54(11):1585-1592, November 1988.

[7] E Chuvieco and R G Congalton. Application of Remote Sensing and GIS to forest fire hazard mapping. Remote Sensing of the Environment, 29:147-159, June 1989.

[8] E Chuvieco and M P Martin. A simple method for fire growth mapping using AVHRR channel 3 data. International Journal of Remote Sensing, 15(16):31413146, 1994.

[9] E Chuvieco and M P Martin. Global fire mapping and fire danger estimation using AVHRR images. Photogrammetric Engineering and Remote Sensing, 60(5):563570, May 1994.

[10] P L Combettes. The foundations of set theoretic estimation. Proceedings of the IEEE, 81(2):182-208, 1993.

[11] S Cubillo E Trillas and J L Castro. Conjunction and disjunction on (0,1). Fuzzy Sets and Systems, 72:155-165, 1995.

[12] E Czogala and J Drewniak. Associative monotic operations in fuzzy set theory. Fuzzy sets and systems, 12:249-269, 1984.

[13] D Dubois and H Prade. A review of Fuzzy Set Aggregation Connectives. Information Sciences, 36:85-121, 1985.

[14] D Dubois and H Prade. Combination of Fuzzy Information in the Framework of Possibility theory. In M A Abidi and R C Gonzalez, editors, Data fusion in Robotics and Machine Intelligence. Academic Press, 1992.

[15] L Garcia and V Caselles. Mapping burns and natural reforestation using thematic mapper data. Geocarto International, 7(1):31-37, 1991.

[16] J D Greer. GIS and Remote Sensing for wildfire suppression and burned area restoration. Photogrammetric Engineering and Remote Sensing, 60(9):1059-1064, September 1994.

[17] J Grunblatt, W K Ottichilo, and R K Sinage. A GIS approach to desertification assessment and mapping. Journal of Arid Environment, 23:81-102, 1992.

[18] M E Jakubauskas, K P Lulla, and W Mausel. Assessment of vegetation change in fire altered landscape. Photogrammetric Engineering and Remote Sensing, 56(3):371377, 1990. 
[19] H Kim and P H Swain. Multisource data analysis in Remote sensing and GIS based on Shafer's theory of evidence. In Proc. of International Geoscience and Remote Sensing Symposium, pages 829-832, 1989.

[20] G J Klir and B Yuan. Fuzzy Sets and Fuzzy Logic, Theory and Applications. Prentice Hall, 1995.

[21] T Lee and J A Richards. Probabilistic and Evidential Approaches for Multisource Data Analysis. IEEE Transactions on Geoscience and Remote Sensing, GE25(3):283-293, May 1987.

[22] B Liberman, A Grankov, A Milshin, S Golovachev, and Valery Vishniakov. Experimental study of fire risk by means of passive microwave and infrared remote sensing methods. In Proc. of International Geoscience and Remote Sensing Symposium, volume 1, pages 175-179, Nebraska, USA, May 1996.

[23] W M Moon. Integration of Geophysical and Geological Data Using Evidential Belief Function. IEEE transactions on Geoscience and Remote Sensing, 28(4):711720, July 1990.

[24] D A Prevedel. A strategic wildfire monitoring package using AVHRR satellite data and GIS . Photogrammetric Engineering and Remote Sensing, 61(3):271-278, March 1995.

[25] D Rokos and P Kolokoussis. The use of Remote Sensing in the evaluation of the Natural Regeneration Potential, Erosion Risk and Desertification Risk after forest fires. In Proc. of International workshop on Remote Sensing and GIS applications to Forest Fire Management, pages 62-65. University of Alcala de Henares, September 1995.

[26] K R Sasikala. Fuzzy Reasoning with Geographic Information System - An aid to decision-making. PhD thesis, School of Elec Eng, IT \& Maths, University of Surrey,, Guildford GU2 5XH, U.K., September 1997.

[27] K R Sasikala, M Petrou, and J Kittler. Fuzzy classification with a GIS as an aid to decision making. EARSeL Advances in Remote Sensing, 4(4):97-105, Nov 1996.

[28] P Smets. Elementary semantic operators. In R R Yager, editor, Fuzzy Set \& Possibility theory: Recent Developments, pages 247-256. Pergamon, 1982.

[29] A Stassopoulou, M Petrou, and J Kittler. Fusion of information and reasoning in a GIS based Decision making system using a Pearl Bayes Network. In The 2nd ACM workshop on advances in GIS, Maryland, USA, pages 128-135, Maryland, December 1994.

[30] A Stassopoulou, M Petrou, and J Kittler. Application of Bayesian and Neural Networks to Geographic Information Processing. In Arthur P.Cracknell, editor, Soft Computing in Remote Sensing Data Analysis, volume 1 of Series in Remote Sensing, pages 65-72. World Scientific, 1996. 
[31] A Stassopoulou, M Petrou and J Kittler. Application of a Bayesian network in a gis based decision making system. International Journal of Geographical Information Sciences, 12:23-45, 1998.

[32] E Trillas C Alsina and L Valverda. On some logical connectives for fuzzy set theory. Journal of Methematical Analysis and its Applications, 93:15-26, 1983.

[33] M J Vasconcelos. Integration of Remote Sensing and GIS for fire risk management. In Proc. of Intl. workshop on Remote Sensing and GIS applications to Forest Fire Management, pages 129-147, University of Alcala de Henares, September 1995.

[34] R R Yager. Multiple objective decision making using fuzzy sets. International Journal of Man-Machine Studies, 9:375-382, 1977.

[35] R R Yager. Concepts, Theory and Techniques, A new methodology for ordinal multi-objective decision based on fuzzy sets. Decision Science, 12:589-600, 1981.

[36] R R Yager. A note on weighted queries in Information Retrieval Systems. Journal of the American Society for Information Science, 38(1):23-24, 1987.

[37] R R Yager. On ordered weighted averaging aggregation operators in multicriteria decision making. IEEE transactions on Systems, Man and Cybernetics, 18(1):183190, 1988.

[38] R R Yager. Connectives and Quantifiers in fuzzy sets. Fuzzy sets and systems, 40:39-75, 1991.

[39] R R Yager and A Kelman. Fusion of Fuzzy Information with Considerations for Compatibility, Partial Aggregation, and Reinforcement. International Journal of Approximate Reasoning, 15(2):93-122, 1996.

[40] L A Zadeh. Fuzzy sets as a basis for theory of possibility. Fuzzy sets and systems, 1(1):3-28, 1978.

[41] J Zhou and D L Civco. Using Genetic Learning Neural Networks for Spatial Decision Making in GIS. Photogrammetric Engineering and Remote Sensing, 62(11):1287-1295, November 1996. 